\title{
Isolated optic nerve gliomas: a multicenter historical cohort study
}

\author{
Ben Shofty, MD, ${ }^{1,2}$ Liat Ben-Sira, MD, ${ }^{1,3}$ Anat Kesler, MD, ${ }^{1,4}$ George Jallo, MD, ${ }^{5}$ \\ Mari L. Groves, MD, ${ }^{5}$ Rajiv R. Iyer, MD, ${ }^{5}$ Alvaro Lassaletta, MD, ${ }^{6}$ Uri Tabori, MD, ${ }^{6}$ Eric Bouffet, MD, ${ }^{6}$ \\ Ulrich-Wilhelm Thomale, MD, ${ }^{7}$ Pablo Hernáiz Driever, MD, ${ }^{7}$ and \\ Shlomi Constantini, MD, MSc, ${ }^{1,8}$ for the Isolated Optic Nerve Abnormalities (IONA) Collaboration
}

1The Gilbert Israeli Neurofibromatosis Center, Dana Children's Hospital; '2Division of Neurosurgery; ${ }^{3 P}$ ediatric Radiology; ${ }^{4}$ Division of Ophthalmology; and ${ }^{8}$ Pediatric Neurosurgery, Dana Children's Hospital, Tel Aviv Medical Center and Tel Aviv University, Tel Aviv, Israel; ${ }^{5}$ Department of Neurosurgery, Johns Hopkins School of Medicine and Hospital, Baltimore, Maryland; ${ }^{6}$ Division of Hematology/Oncology, The Hospital for Sick Children, Toronto, Ontario, Canada; and ${ }^{7}$ Pediatric Neurosurgery, Charité Universitätsmedizin, Campus Virchow Klinikum, Berlin, Germany

OBJECTIVE Isolated optic nerve gliomas (IONGs) constitute a rare subgroup of optic pathway gliomas (OPGs). Due to the rarity of this condition and the difficulty in differentiating IONGs from other types of OPGs in most clinical series, little is known about these tumors. Currently, due to lack of evidence, they are managed the same as any other OPG.

METHODS The authors conducted a multicenter retrospective cohort study aimed at determining the natural history of IONGs. Included were patients with clear-cut glioma of the optic nerve without posterior (chiasmatic/hypothalamic) involvement. At least 1 year of follow-up, 2 MRI studies, and 2 neuro-ophthalmological examinations were required for inclusion.

RESULTS Thirty-six patients with 39 tumors were included in this study. Age at diagnosis ranged between 6 months and 16 years (average 6 years). The mean follow-up time was 5.6 years. Twenty-five patients had neurofibromatosis Type 1. During the follow-up period, $59 \%$ of the tumors progressed, $23 \%$ remained stable, and $18 \%$ (all with neurofibromatosis Type 1) displayed some degree of spontaneous regression. Fifty-one percent of the patients presented with visual decline, of whom $90 \%$ experienced further deterioration. Nine patients were treated with chemotherapy, 5 of whom improved visually. Ten patients underwent operation, and no local or distal recurrence was noted.

CONCLUSIONS Isolated optic nerve gliomas are highly dynamic tumors. Radiological progression and visual deterioration occur in greater percentages than in the general population of patients with OPGs. Response to chemotherapy may be better in this group, and its use should be considered early in the course of the disease.

https://thejns.org/doi/abs/10.3171/2017.6.PEDS17107

KEY WORDS optic nerve; optic pathway glioma; NF1; neurofibromatosis Type 1; oncology

$\mathrm{T}$ HIS is the first study focused on isolated optic nerve gliomas (IONGs). Today, there remains a debate as to best practices regarding the management of IONGs. The present study sheds light on these rare lesions, and suggests that they are highly active tumors that tend toward radiological progression. In addition, they tend to cause visual deterioration in higher percentages than do general optic pathway gliomas (OPGs). Chemotherapy may contribute to preserved vision in this group, and should be considered early. This study will aid physicians caring for patients with IONGs in better risk stratification, and in determining the best treatment course.

Optic pathway gliomas are a heterogeneous group of low-grade tumors that are centered in the visual neural

ABBREVIATIONS IONG = isolated optic nerve glioma; NF1 = neurofibromatosis Type 1; ON = optic nerve; ONSD = ON sheath dilation; OPG = optic pathway glioma. SUBMITTED February 25, 2017. ACCEPTED June 14, 2017. 
structures, from the optic nerve (ON) anteriorly to the optic radiations posteriorly. ${ }^{24}$ The OPGs, traditionally associated with childhood and neurofibromatosis Type 1 (NF1), ${ }^{14}$ are infamous for being a complex, heterogeneous group of tumors. Predicting outcome and prognosis for patients with OPGs is usually challenging..$^{16}$ The vast amount of heterogeneity of OPGs is caused by a variety of factors, including the following: age at presentation; sex; coexistence of NF1; tumoral genetic variability (such as $B R A F$ mutation or fusion); anatomical architecture of the tumor (cystic or solid); associated clinical manifestations (such as diencephalic syndrome); and extension along the optic pathways and external to them (intraorbital, chiasmatic/hypothalamic, posterior pathways, dissemination into subarachnoid spaces, and so on). ${ }^{21}$ All of these factors significantly complicate patient stratification in the clinical setting, clinical research of natural history, and categorization of responses to various treatment modalities. Prognostic factors for progression and visual deterioration, as described in the literature, include the following: age at presentation; tumor location; tumor volume; presence of diencephalic syndrome; fractional anisotropy values as measured with diffusion tensor MRI; and others. ${ }^{8,10,17}$

Most of the recent case series describing OPGs deal with an anatomically and morphologically diverse group of tumors, and therefore any general conclusions drawn from these series may be misleading. Although in some clinical series tumor location appears to have prognostic significance, it is unclear if tumors that are centered at various locations, such as the $\mathrm{ON}$ or optic radiations, actually differ in their biological behavior due to their location, or if they could be equally well prognosticated using other accepted factors. In addition, many of these tumors are quite large, and although centered on the chiasm, they can also include extensive involvement of the $\mathrm{ON}$ or posterior pathways. Therefore, in most cases it is not possible to establish definitively which part of the tumor is responsible for visual deterioration. Because most series show no correlation between radiological progression and visual status, there is a need for a methodology that would allow the identification and characterization of tumor subgroups. ${ }^{6,11,15,21}$

Isolated optic nerve gliomas are not well studied. These lesions, which are restricted to the $\mathrm{ON}$, without extension to the chiasm or posteriorly, are traditionally lumped together with chiasmatic tumors or other optic pathway involvement. Two major series have focused on ON gliomas in a mixed population of patients with and without NF1. The first study, presented by Tow et al. ${ }^{25}$ described a series of 16 patients with a total of 19 tumors, some of them collected from the pre-MRI era. This series, with an impressive follow-up time of 15 years, demonstrated a high percentage of treated patients (10/16), and a rather poor visual outcome. The second series, presented by Zeid et al. ${ }^{28}$ was not as homogeneous as the first series -6 of their 12 patients had chiasmatic involvement, in addition to the affected $\mathrm{ON}$.

This current, multicenter, retrospective cohort study is aimed at ascertaining and describing the natural history and visual outcome of patients with IONGs.

\section{Methods}

This study was approved by the local institutional review board at each of the participating centers (Tel Aviv Medical Center; Hospital for Sick Kids Toronto; Johns Hopkins Medical Center Baltimore; and Charité Universitätsmedizin Berlin). Data were accrued retrospectively from 1990 to 2016. Each center entered its data into an electronic database following complete anonymization of the patients. Databases and representative images were sent to the Tel Aviv Medical Center for centralized analysis.

Included were all patients (NF1 and sporadic) followed at different centers who had an enlarged $\left(>4 \mathrm{~mm}^{4}\right)$, enhancing IONG (intracranial involvement not reaching the chiasm; bilateral tumors were allowed), with at least 1 year of follow-up (both radiological and clinical). Excluded were patients with posterior optic pathway involvement (Dodge Classes II and III ${ }^{9}$ ), hydrocephalus, any demyelinating or inflammatory disease of the CNS, and any known active brain tumor outside of the optic pathway. Diffuse, NF1-related changes (T2 hyperintensities, and so on), ON tortuosity, ${ }^{1}$ and ON sheath dilation (ONSD) ${ }^{19}$ were not exclusion criteria. Visual deterioration was defined as a drop of 2 lines in the Snellen test and/or worsening in a visual field test. Visual decline was defined as visual deterioration caused directly or indirectly by the tumor, after ruling out other unrelated causes by examination with optimal refraction correction that was documented by the treating neuro-ophthalmologist.

Representative imaging at diagnosis and at last followup for each patient was reviewed. Fulfillment of the inclusion criteria was verified for each subject. Prechiasmatic ON involvement was differentiated from chiasmatic based on MRI (coronal thin sections). When any part of the chiasm was involved, either by signal abnormalities (T2-weighted sequences) or enhancement (T1-weighted sequences with gadolinium), the patient was excluded from this study. Radiologically, the tumor's location was determined (intraorbital, prechiasmatic, or both), and other abnormalities such as ON thickness and ONSD were documented. Radiological progression was determined according to the medical record and official MRI interpretation as provided by each center, because no clear criteria exist in the literature regarding IONG tumor progression. For the comparison of averages between groups we used the Student t-test.

\section{Results}

Thirty-six patients with 39 tumors at presentation (3 patients with bilateral tumors) were accrued over the study period. Two patients who presented with nerve thickening developed IONGs during the follow-up period and were added to the study, for a total of 38 patients. Demographic data and breakdown for each center are shown in Table 1.

The mean age at diagnosis for the entire cohort was 5.6 years (median 4.3 years, range 6 months -16 years). Of a total of 38 patients, 20 were female, and 25 had NF1. Age was not associated with either radiological or visual outcome ( $\mathrm{p}>0.5$ for both). The mean radiological follow-up was $5.8 \pm 0.6$ years, and the mean ophthalmological fol- 
TABLE 1. Breakdown of the patient population with IONGs for each of the participating centers

\begin{tabular}{lcccc}
\hline Center & No. of Patients & Sex & $\%$ w/ NF1 (no.) & Average Age (yrs) \\
\hline TAMC & 10 & $7 \mathrm{~F} / 3 \mathrm{M}$ & $100 \%(10)$ & 4.7 \\
\hline JHMC & 7 & $3 \mathrm{~F} / 4 \mathrm{M}$ & $14 \%(1)$ & 8.6 \\
\hline SKH & 16 & $8 \mathrm{~F} / 8 \mathrm{M}$ & $62 \%(10)$ & 5.6 \\
\hline CUB & 5 & $2 \mathrm{~F} / 3 \mathrm{M}$ & $80 \%(4)$ & 3.1
\end{tabular}

CUB = Charité Universitätsmedizin Berlin; JHMC = Johns Hopkins Medical Center; SKH = Sick Kids Hospital; TAMC = Tel Aviv Medical Center.

low-up was $5.6 \pm 0.5$ years (mean values are given $\pm \mathrm{SD}$ ). Three patients had bilateral disease at presentation; all of them had NF1. In 18 cases there was isolated intraorbital involvement; in 9 there was isolated intracranial, prechiasmatic involvement; and in 12 both parts of the nerve were involved (Fig. 1).

The mean ON width at presentation (as measured on a fat-suppressed T1-weighted sequence obtained with contrast) was $10.4 \pm 4 \mathrm{~mm}$. No difference in average ON width was found between patients with NF1 and sporadic IONGs $(p=0.6)$. Patients who suffered from visual deterioration had a larger $\mathrm{ON}$ width $(11.4 \pm 4.7 \mathrm{~mm})$ than did patients with stable vision $(8.3 \pm 3.9 \mathrm{~mm}, \mathrm{p}=0.06)$. During the follow-up period, 23 tumors (59\%) demonstrated radiological progression (Table 2).

Of the 25 patients with NF1 patients who had IONGs, $15(60 \%)$ experienced radiological progression. Seven of the 25 patients with NF1 (28\%) demonstrated some spontaneous regression. No patient with a sporadic IONG demonstrated spontaneous regression. Nineteen (50\%) patients (19 eyes) presented with visual impairment, 17 of whom experienced further deterioration during the follow-up period (Fig. 2).

An additional 7 eyes (of 20) that had normal vision at presentation experienced visual decline during the followup period, for a total of 24 eyes $(61 \%)$ that deteriorated. All 9 of the patients with NF1 who presented with impaired vision experienced further deterioration during the study period. Of 16 patients with NF1 who presented with normal vision, in 7 (43\%) vision deteriorated.

\section{No Tumor at Presentation}

Twelve patients (from the entire cohort, all with NF1) presented with a thickening of their contralateral $\mathrm{ON}$ that did not qualify as an IONG. Of this group, in 6 patients (50\%) the lesion progressed to a tumor, 4 patients remained stable, and 2 patients demonstrated regression during the follow-up period. In 3 other patients with NF1, we noticed a progression from a normal-appearing nerve to an IONG during the follow-up period. Of the 6 patients in whom the lesion progressed from $\mathrm{ON}$ thickening to an IONG, 4 experienced visual decline. Of the 3 patients with a normal nerve that progressed to an IONG, only 1 experienced visual decline. One patient (without NF1) had progression from an IONG to a Dodge Class II tumor; he developed chiasmal involvement during the follow-up period. However, this patient's tumor extended posteriorly all the way to the edge of the chiasm. Although this patient's lesion
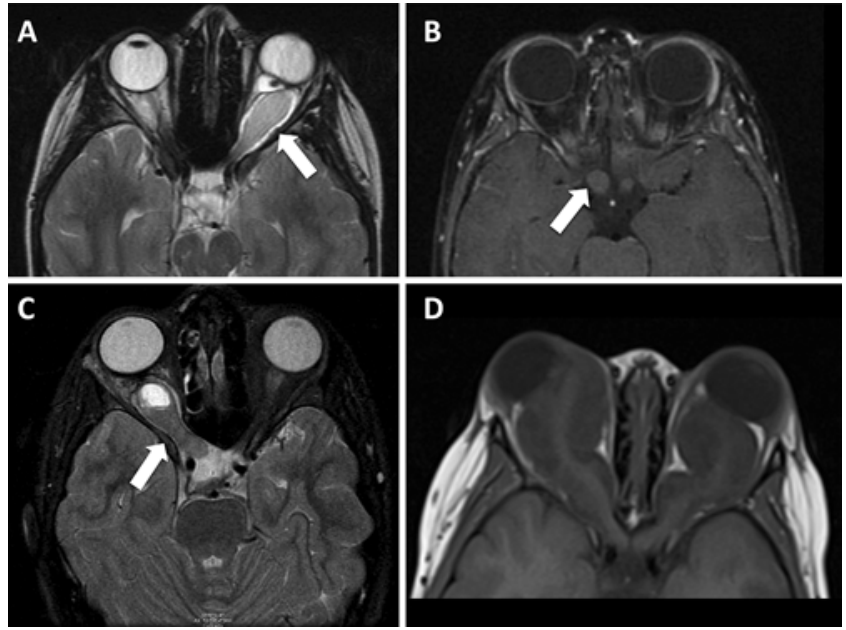

FIG. 1. Axial MR images demonstrating anatomical classifications of IONGs (arrows). A: Intraorbital tumor. B: Prechiasmatic, intracranial tumor with no intraorbital involvement. C: Entire ON involved. D: Bilateral ON involvement.

represented a borderline IONG from the outset, he was included because his disease met our inclusion criteria of tumors restricted to the nerve without frank changes within the chiasm on MRI.

\section{Treated Patients}

Treatment modalities used included chemotherapy (9 patients received vincristine and carboplatin, all due to visual deterioration -4 with concomitant radiological progression); radiation (2 patients); and $\mathrm{ON}$ resection (10 patients). In the NF1 subpopulation, 5 patients (of the total of 9 treated patients) received chemotherapy and 3 underwent surgery. Of the patients treated with chemotherapy, 5 benefited visually (Table 2), although none of them demonstrated significant long-term radiological response. One patient treated with chemotherapy demonstrated significant radiological response that was not associated with visual improvement (Fig. 3).

Of the patients who underwent resection of the affected nerve, with or without resection of the globe $(n=10)$, all had progressive tumors and had severe visual impairment or total blindness in the affected eye prior to surgery. Following resection and during the follow-up period, there

TABLE 2. Radiological-visual correlation in 39 IONGs

\begin{tabular}{lcccc}
\hline Radiological & \multicolumn{4}{c}{ Overall Visual Outcome } \\
\cline { 2 - 5 } Tumor Behavior & Improved & Stable & Decline & Total \\
\hline Regressed & 0 & 5 & 2 & 7 \\
\hline Stable & 1 & 4 & 4 & 9 \\
\hline Progressed & 4 & 5 & 14 & 23 \\
\hline Total & 5 & 14 & 20 & 39 \\
\hline
\end{tabular}

Note that all patients with improved vision received chemotherapy. Four of them initially experienced visual deterioration and subsequently improved following chemotherapy. One patient received chemotherapy for radiological progression and demonstrated radiological response despite significant visual decline. 


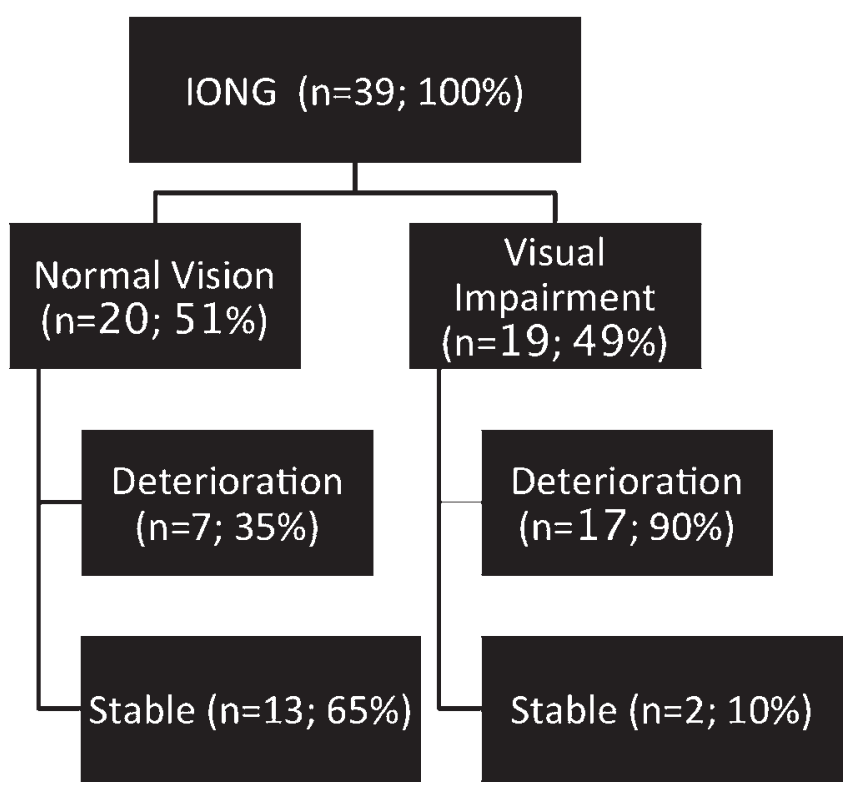

FIG. 2. Chart showing the visual outcome of the entire cohort of patients with IONGs.

was no further development of other tumors. No leptomeningeal dissemination or progression to the chiasm was noted following surgery. Information regarding microscopic involvement of the resection margins was not available.

In 2 patients (non-NF1), tumors were irradiated due to progressive visual loss. Neither patient benefited from this treatment, visually or radiologically.

\section{Discussion}

This is the largest study focusing on the natural history and response to treatment of IONGs in the MR era. Despite the fact that OPGs comprise approximately $5 \%$ of pediatric brain tumors, and that $30 \%$ of patients with NF1 will suffer from such a tumor, this unique group has not been properly addressed to date. This may be due to the relative rarity of the IONG subset, or the exclusion of this group from SIOP and COG (Societé Internationale d'Oncologie Pédiatrique and Children's Oncology Group) clinical studies. To recruit a meaningful number of patients, we joined forces with a total of 5 centers, all of them with a significant neurofibromatosis program and a strong pediatric neuro-oncological orientation. Based on this study, it is evident that IONGs are highly active tumors with a significantly higher percentage of progression compared with chiasmatic tumors. In addition, they are associated with significant and progressive visual impairment.

In 2003, Tow et al. published a mixed series of 47 patients with anterior OPGs. ${ }^{25}$ Of this group, 16 patients had IONGs. Fifty percent of their patients with IONGs were symptomatic at diagnosis, and $53 \%$ had significantly decreased vision in the affected eye. In that report, 6 patients underwent resection and in 4 the tumors were irradiated (only 1 patient who underwent irradiation improved). No chemotherapy was used at that time. These findings, in line with our current report, suggest that these tumors are highly active both visually and radiologically and may lead to visual loss; 11/16 (68\%) patients in the group reported by Tow et al. had no light perception at last followup. In our series, interestingly, visual deterioration was noted in $61 \%$, whereas radiological progression was evident in $38 \%$, demonstrating once again the lack of clinicalradiological correlation in OPGs and IONGs.

Interestingly, in our series an IONG's maximal width was not associated with radiological progression; however, a trend toward association with visual deterioration was noted. Despite scans that indicate no radiological progression, these patients (tumor $>8 \mathrm{~mm}$ ) should be closely followed, because they may be at increased in risk of visual deterioration.

Other than this series, reports dealing with IONGs tend to be sporadic, mainly presenting case studies. One recent report described 2 patients with IONGs in whom chemotherapy failed and who then underwent a biopsy. ${ }^{18}$ In both cases pathology was consistent with WHO Grade I pilocytic astrocytoma. In both cases, significant visual decline due to vascular injury was evident following biopsy. This report further emphasizes the current recommendations to avoid biopsy in OPGs, and especially in IONGs. The role of surgery in IONGs is still controversial. A recent report discusses the surgical option in a patient with a blind eye, proptosis, and pain. ${ }^{5}$ Surgical removal of a growing mass within the orbit in a blind eye is usually effective in addressing proptosis and pain. The more controversial dilemma is whether resection of the nerve with margins posterior to the affected part is indicated to avoid slow progression of the tumor from the nerve to the chiasm. Although this argument is mentioned occasionally, resection of the nerve has never been shown to have an oncological impact. The only patient in our series who seemed to display progression from the nerve to the chiasm had a tumor that was already touching the chiasm at presentation; therefore prophylactic resection with margins was not an option. Posterior progression within the nerve into the chiasm was not documented in any of the other patients in our series. This important finding could represent a strong argument against the performance of a prophylactic procedure based solely on oncological considerations.

In our series, $25.6 \%$ of the patients underwent $\mathrm{ON}$ resection, all of them with pilocytic astrocytoma, with no local or distal recurrence evident during $>5$ years of follow-up. No progression to the chiasm was seen in the nonsurgically treated group either. This finding highlights the surgical dilemma: do we need to consider ON resection for IONGs to prevent distal progression? This progression, sometimes seen in other types of OPG and other juvenile pilocytic astrocytomas, ${ }^{12}$ was not evident in our series. Another interesting question that is beyond the scope of this study is the possibility of 2 independent ON tumors occurring without macroscopic chiasmal involvement, or 1 macroscopic tumor affecting both ONs and microscopically involving the chiasm. Another surgical option that may be valid, although it was not used in any of our centers, is optic nerve sheath fenestration for tumor-associated ONSD. In a single case report, Vanderveen et al. report visual stabilization and even improvement following nerve sheath decompression. ${ }^{26}$ This approach needs 

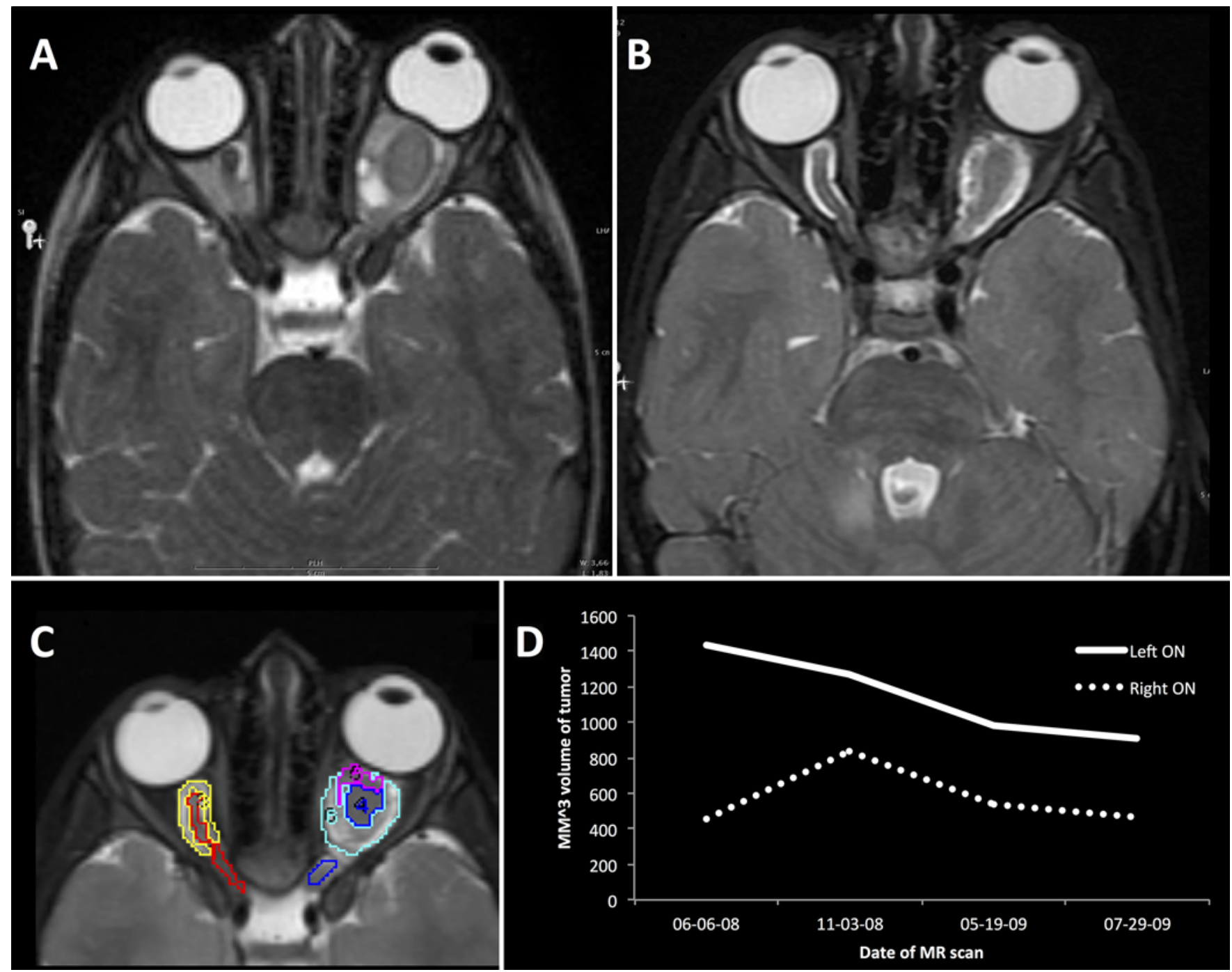

FIG. 3. A 27-month-old girl presented with 1 month of painful proptosis and visual decline in the left eye. On presentation, a clinical diagnosis of NF1 was made. Axial MR images obtained at diagnosis revealed a large, 15-mm IONG on the left, and ON thickening with sleeve dilation on the right (A). Prompt treatment with vincristine and carboplatin was initiated. Over the treatment period a significant response was noted (B). Volumetric follow-up for the nerves was used to estimate treatment efficacy (C). In brief, following coregistration of each scan and of T1- and T2-weighted sequences, the nerve was segmented semiautomatically (see Zeid et al. for a detailed description of our method). Volumetric results during the follow-up period (4 scans) are shown (D). Unfortunately, despite significant radiological response, her vision deteriorated to the point of no light perception on the left side. Figure is available in color online only.

further exploration and validation, but may be considered in early cases with significant tumor-associated ONSD.

Another important issue that arises from our series is the development of IONGs from precursor radiological abnormalities. In patients with NF1, many radiological abnormalities of the $\mathrm{ON}$ are commonly present, such as T2 signal changes, ONSD, focal nerve enhancement, ON tortuosity, and so on. A recent report by Levin et al. examined the risk for optic nerve glioma development in patients with $\mathrm{ON}$ abnormalities. In their series, patients with NF1 who had a tortuous ON were more likely to develop an OPG, but ONSD or nerve thickening were not associated with an increased risk for tumor development..$^{13}$ In our series of IONGs, we encountered 12 patients with NF1 who had contralateral nerve thickening. In $50 \%$ of the patients with contralateral $\mathrm{ON}$ thickening the lesion progressed to an IONG, and the patient suffered visual decline. In addition, some patients with NF1 demonstrated progression from an initially normal-appearing nerve. Due to the retrospective nature of this study, selection of these patients is highly biased and may not represent the entire NF1 or OPG population. Regardless, in a patient with an OPG or IONG, a thickened contralateral nerve should be closely followed.

Medical treatment options for IONGs were previously discussed by Tow et al. Our study cannot provide specific treatment recommendations due to the limited number of treated patients. It should, however, be noted that in both patients treated with radiation, no beneficial effect was observed, either visual or radiological. Chemotherapy dem- 
onstrated limited radiological impact (1 of 9 patients), but a significant visual impact was noted, with 5 of 9 patients (1 of 5 with NF1) demonstrating clinical improvement following treatment. This percentage is higher than the accepted response rate found in the literature $(14 \%-32 \%)$ following first-line treatment for general OPGs., ${ }^{71,20}$ This finding potentially indicates a need for better patient selection and accurate timing of treatment. Our data suggest that it may be reasonable to recommend that high-risk patients with IONGs who present with visual impairment receive early chemotherapy, because without treatment vision will continue to deteriorate in $90 \%$ of these patients. Because the main aim of chemotherapy is visual improvement or stabilization, which is often difficult to assess in young children,,$^{2,3}$ there is a need for improved modalities of radiological follow-up. Volumetric analyses of the ON, as presented in Fig. 3, are not trivial. Unlike in chiasmatic/ hypothalamic OPGs, where they are rapidly becoming the standard of care, ${ }^{22,23,27}$ volumetrics for IONGs require specific, time-consuming sequences that are not widely available.

\section{Conclusions}

Isolated optic nerve gliomas are highly active tumors that tend toward radiological progression, and cause visual deterioration in higher percentages of patients than do general OPGs. Chemotherapy may contribute to preservation of vision in this group, and deserves further study. Surgical treatment provides excellent local and distal control. Further examination of these unique tumors via prospective trials is advised.

\section{Acknowledgments}

We thank Ms. Edna Fadida for coordinating this multicenter study.

\section{References}

1. Armstrong GT, Localio AR, Feygin T, Bilaniuk L, Phillips PC, Fisher MJ, et al: Defining optic nerve tortuosity. AJNR Am J Neuroradiol 28:666-671, 2007

2. Avery RA, Bouffet E, Packer RJ, Reginald A: Feasibility and comparison of visual acuity testing methods in children with neurofibromatosis type 1 and/or optic pathway gliomas. Invest Ophthalmol Vis Sci 54:1034-1038, 2013

3. Avery RA, Ferner RE, Listernick R, Fisher MJ, Gutmann DH, Liu GT: Visual acuity in children with low grade gliomas of the visual pathway: implications for patient care and clinical research. J Neurooncol 110:1-7, 2012

4. Avery RA, Mansoor A, Idrees R, Biggs E, Alsharid MA, Packer RJ, et al: Quantitative MRI criteria for optic pathway enlargement in neurofibromatosis type 1. Neurology 86:2264-2270, 2016

5. Borghei-Razavi H, Shibao S, Schick U: Prechiasmatic transection of the optic nerve in optic nerve glioma: technical description and surgical outcome. Neurosurg Rev 40:135-141, 2017

6. Campagna M, Opocher E, Viscardi E, Calderone M, Severino SM, Cermakova I, et al: Optic pathway glioma: long-term visual outcome in children without neurofibromatosis type-1. Pediatr Blood Cancer 55:1083-1088, 2010

7. Dalla Via P, Opocher E, Pinello ML, Calderone M, Viscardi E, Clementi M, et al: Visual outcome of a cohort of children with neurofibromatosis type 1 and optic pathway glioma fol- lowed by a pediatric neuro-oncology program. Neuro Oncol 9:430-437, 2007

8. de Blank PM, Berman JI, Liu GT, Roberts TP, Fisher MJ: Fractional anisotropy of the optic radiations is associated with visual acuity loss in optic pathway gliomas of neurofibromatosis type 1. Neuro Oncol 15:1088-1095, 2013

9. Dodge HW Jr, Love JG, Craig WM, Dockerty MB, Kearns TP, Holman CB, et al: Gliomas of the optic nerves. AMA Arch Neurol Psychiatry 79:607-621, 1958

10. Dodgshun AJ, Elder JE, Hansford JR, Sullivan MJ: Longterm visual outcome after chemotherapy for optic pathway glioma in children: site and age are strongly predictive. Cancer 121:4190-4196, 2015

11. Fisher MJ, Balcer L, Gutmann DH, Listernick R, Ferner R, Packer R, et al: Neurofibromatosis type I associated optic glioma visual outcomes following chemotherapy: an international multi-center retrospective analysis. Neuro Oncol 12:ii19-ii20, 2010 (Abstract)

12. Gil Z, Beni-Adani L, Siomin V, Nagar H, Dvir R, Constantini S: Ascites following ventriculoperitoneal shunting in children with chiasmatic-hypothalamic glioma. Childs Nerv Syst 17:395-398, 2001

13. Levin MH, Armstrong GT, Broad JH, Zimmerman R, Bilaniuk LT, Feygin T, et al: Risk of optic pathway glioma in children with neurofibromatosis type 1 and optic nerve tortuosity or nerve sheath thickening. Br J Ophthalmol 100:510-514, 2016

14. Listernick R, Louis DN, Packer RJ, Gutmann DH: Optic pathway gliomas in children with neurofibromatosis 1: consensus statement from the NF1 Optic Pathway Glioma Task Force. Ann Neurol 41:143-149, 1997

15. Moreno L, Bautista F, Ashley S, Duncan C, Zacharoulis S: Does chemotherapy affect the visual outcome in children with optic pathway glioma? A systematic review of the evidence. Eur J Cancer 46:2253-2259, 2010

16. Nicolin G, Parkin P, Mabbott D, Hargrave D, Bartels U, Tabori U, et al: Natural history and outcome of optic pathway gliomas in children. Pediatr Blood Cancer 53:1231-1237, 2009

17. Opocher E, Kremer LC, Da Dalt L, van de Wetering MD, Viscardi E, Caron HN, et al: Prognostic factors for progression of childhood optic pathway glioma: a systematic review. Eur J Cancer 42:1807-1816, 2006

18. Revere KE, Katowitz WR, Katowitz JA, Rorke-Adams L, Fisher MJ, Liu GT: Childhood optic nerve glioma: vision loss due to biopsy. Ophthal Plast Reconstr Surg 33 (3S Suppl 1):S107-S109, 2017

19. Shofty B, Ben-Sira L, Constantini S, Freedman S, Kesler A: Optic nerve sheath diameter on MR imaging: establishment of norms and comparison of pediatric patients with idiopathic intracranial hypertension with healthy controls. AJNR Am J Neuroradiol 33:366-369, 2012

20. Shofty B, Ben-Sira L, Freedman S, Yalon M, Dvir R, Weintraub M, et al: Visual outcome following chemotherapy for progressive optic pathway gliomas. Pediatr Blood Cancer 57:481-485, 2011

21. Shofty B, Ben-Sira L, Kesler A, Constantini S: Optic pathway gliomas. Adv Tech Stand Neurosurg 42:123-146, 2015

22. Shofty B, Mauda-Havakuk M, Weizman L, Constantini S, Ben-Bashat D, Dvir R, et al: The effect of chemotherapy on optic pathway gliomas and their sub-components: a volumetric MR analysis study. Pediatr Blood Cancer 62:1353-1359, 2015

23. Shofty B, Weizman L, Joskowicz L, Constantini S, Kesler A, Ben-Bashat D, et al: MRI internal segmentation of optic pathway gliomas: clinical implementation of a novel algorithm. Childs Nerv Syst 27:1265-1272, 2011

24. Shuper A, Horev G, Kornreich L, Michowiz S, Weitz R, Zaizov R, et al: Visual pathway glioma: an erratic tumour with therapeutic dilemmas. Arch Dis Child 76:259-263, 1997 
25. Tow SL, Chandela S, Miller NR, Avellino AM: Long-term outcome in children with gliomas of the anterior visual pathway. Pediatr Neurol 28:262-270, 2003

26. Vanderveen DK, Nihalani BR, Barron P, Anderson RL: Optic nerve sheath fenestration for an isolated optic nerve glioma. $\mathbf{J}$ AAPOS 13:88-90, 2009

27. Weizman L, Ben Sira L, Joskowicz L, Constantini S, Precel R, Shofty B, et al: Automatic segmentation, internal classification, and follow-up of optic pathway gliomas in MRI. Med Image Anal 16:177-188, 2012

28. Zeid JL, Charrow J, Sandu M, Goldman S, Listernick R: Orbital optic nerve gliomas in children with neurofibromatosis type 1. J AAPOS 10:534-539, 2006

\section{Disclosures}

The authors report no conflict of interest concerning the materi- als or methods used in this study or the findings specified in this paper.

\section{Author Contributions}

Conception and design: Constantini, Shofty, Bensira, Kesler, Iyer, Lassaletta, Tabori, Bouffet, Thomale, Hernáiz Driever. Acquisition of data: Shofty, Jallo, Groves, Lassaletta, Hernáiz Driever. Analysis and interpretation of data: Constantini, Shofty, Bensira. Drafting the article: Constantini, Shofty. Critically revising the article: Constantini, Shofty, Kesler, Jallo, Groves, Iyer, Lassaletta, Tabori, Bouffet, Thomale, Hernáiz Driever. Reviewed submitted version of manuscript: Shofty. Statistical analysis: Shofty. Study supervision: Constantini.

\section{Correspondence}

Shlomi Constantini, Department of Pediatric Neurosurgery, Dana Children's Hospital, Tel Aviv Medical Center, 6 Weizmann St., Tel Aviv 64239, Israel. email: sconsts@netvision.net.il. 